\title{
Análise da produção científica a respeito de psicoterapia analítica funcional (FAP)
}

\section{Analysis of scientific production on functional analytic psychotherapy (FAP) Análisis de la producción científica en psicoterapia analítica funcional (FAP)}

\author{
Alex Sandro Ribeiro', Susana Rodrigues de Oliveira², Nicodemos Batista Borges ${ }^{3}$
}

Resumo: O presente estudo visou caracterizar e discutir a produção científica a respeito de FAP, mais especificamente estender o estudo de Mangabeira et al. (2012) para a produção compreendida entre 2010 e 2013. O método consistiu em levantar e analisar artigos sobre FAP (a) indexados em cinco bases de dados, a saber: PubMed, SciELO, Virtual Health Library - Psychology, MEDLINE e Lilacs; (b) listados nas referências do site de FAP http://functionalanalyticpsychotherapy.com, bem como nos sites Science Direct e ISI Web of Knowledge; e (c) publicados nos jornais JABA, JEAB, The Behavior Analyst, The Behavior Analysis Today, IJBCT e JEIBI. A palavra-chave utilizada foi "Functional Analytic Psychotherapy", considerando os idiomas inglês e português, e a sigla "FAP". Os 46 estudos encontrados foram categorizados conforme suas características bibliográficas, metodológicas e temáticas. Os principais resultados encontrados foram que: houve um aumento na quantidade de publicações a respeito de FAP no último triênio, quando comparada aos períodos anteriores - resultado este que pode ter sido influenciado pela edição de um volume especial do periódico The International Journal of Behavioral Consultation and Therapy dedicado ao tema -; a maioria dos estudos são de campo/empíricos e lançam mão de medidas de observação para coleta de dados; há uma preferência por estudos com poucos participantes (sugerindo o uso de delineamentos de sujeito como seu próprio controle) e com população adulta; e os principais temas estudos têm sido tratamento com FAP, comparação com ACT e terapia de grupo com FAP.

Palavras-chave: Psicoterapia analítica funcional, FAP, revisão de literatura, análise da produção científica. 
Abstract: This study aimed to revise the scientific production of Functional Analytic Psychotherapy (FAP). The method consisted on surveying and analysing FAP related articles which were either (a) indexed on the following five databases: PubMed, SciELO, Virtual Health Library - Psychology, MEDLINE and Lilacs; (b) listed on the references of the website http:// functionalanalyticpsychotherapy.com, as well as of the websites Science Direct and ISI Web of Knowledge; and (c) published at the journals JABA, JEAB, The Behavior Analyst, The Behavior Analysis Today, IJBCT and JEIBI. The keyword used was "Functional Analytic Psychotherapy", considering both English and Portuguese languages, and the acronym "FAP". The 46 studies found were categorized according to their bibliographic, methodological and thematic characteristics. The main results found were that: there was an increase in the number of publications about FAP in the past triennium if compared with previous periods - which may have been influenced by special edition of the The International Journal of Behavioral Consultation and Therapy devoted to the subject; most of the studies are empirical/field studies and make use of observation tools for data collection; there is a preference for studies with fewer participants (suggesting the use of single-subject designs) and with adult population; and the main topics studied were treatments using FAP, comparison with ACT and group therapies with FAP

Keywords: Functional Analytic Psychotherapy, FAP, literature review, scientific production analysis.

Resumen: Este estudio tuvo como objetivo caracterizar y analizar la producción científica sobre FAP, más específicamente ampliar el estudio de Mangabeira et al. (2012) para la producción hecha entre 2010 y 2013. El método consistió en la compilación y análisis de artículos sobre la FAP (a) indexados en cinco bases de datos, a saber: PubMed, SciELO, Virtual Health Library - Psychology, MEDLINE y LILACS; (b) que figuran en las referencias del sitio FAP http://functionalanalyticpsychotherapy.com, y de los sitios web Science Direct e ISI Web of Knowledge; y (c) publicados en los periódicos JABA, JEAB, The Behavior Analyst, The Behavior Analysis Today, IJBCT y JEIBI. La palabra clave utilizada fue "Functional Analytic Psychotherapy", teniendo en cuenta el portugués y el inglés, y el acrónimo "FAP". Los 46 estudios encontrados se clasificaron de acuerdo a su literatura, metodológico y características temáticas. Las principales conclusiones fueron que: hubo un aumento en el número de publicaciones sobre la FAP en los últimos tres años, en comparación con períodos anteriores - un resultado que puede haber sido influenciado por la edición de un número especial de la revista The International Journal of Behavioral Consulta y Terapia en el tema -; la mayoría de los estudios son de campo / empíricos y utilizan medidas de observación para la recolección de datos; hay una preferencia por los estudios con pocos participantes (que sugieren el uso de contornos del sujeto como su propio control) y con población adulta; y los principales temas de estudio han sido los tratamientos con PAF, comparación con ACT y terapia de grupo con FAP.

Palabras-clave: Psicoterapia Analítica Funcional, FAP, revisión de la literatura, análisis de la producción científica. 
Kohlenberg e Tsai (1991/2001), baseados nos princípios do Behaviorismo Radical, propuseram uma forma de intervenção clínica que privilegia a análise e intervenção diretamente sobre os comportamentos do cliente, por meio da relação terapêutica (Borges, 2009; Conte \& Brandão, 2012). Esse procedimento foi chamado pelos autores de Psicoterapia Analítica Funcional (FAP) e tornou-se um tipo de intervenção frequentemente adotado por clínicos analítico-comportamentais, pois permite que o profissional utilize a relação terapeuta-cliente como instrumento de intervenção e como veículo através do qual a mudança possa ocorrer (Borges, 2009; Greben, 1981; Wielenska, 2012), não restringindo a terapia a intervenções por meio de comportamento verbalmente controlados.

De acordo com seus proponentes, Kohlenberg e Tsai (1991/2001), "a FAP tem sua base na investigação de como o reforçamento, a especificação de comportamentos clinicamente relevantes e a generalização podem ser obtidos dentro das limitações de uma situação típica de tratamento de consultório" (p. 9). Enquanto procedimento de intervenção, a FAP se aplica tanto a comportamentos que ocorrem naturalmente na relação entre terapeuta e cliente, como aqueles que podem ser estrategicamente evocados pelo clínico (Wielenska, 2012).

A FAP é procedimento terapêutico de grande utilidade para intervir sobre uma gama de comportamentos socialmente problemáticos, como: medos, dificuldades em expressar sentimentos, hostilidade, hipersensibilidade a críticas, ansiedade social, comportamentos obsessivos-compulsivos, etc. Para o uso deste procedimento, é necessário apenas que os comportamentos-alvo ocorram ou possam ser evocados durante a(s) sessão(ões) (Kohlenberg \& Tsai, 1991/ 2001), podendo ser diretamente consequenciados.

A esses comportamentos do cliente que ocorrem durante a sessão e têm relação com o problema, dá-se o nome de comportamentos clinicamente relevantes ou no inglês, como é mais comum, clinically relevant behaviors (CRB) (Kohlenberg \& Tsai, 1991/2001). Os comportamentos-alvo apresentados em sessão que deveriam diminuir de ocorrência no processo terapêutico são chamados de CRB1. Os CRB2 são os comportamentos de melhora do cliente, e devem aumentar de frequência ou intensidade.
Já os CRB3 são explicações que o cliente faz de seu comportamento, algumas vezes acompanhadas de relatos de efetiva mudança ocorrida fora do consultório (Taccola, 2007; Wielenska, 2012).

Para que o clínico possa modificar os comportamentos do cliente em sessão, deve-se estar sob controle de cinco regras (Kohlenberg \& Tsai, 1991/2001), sendo elas: (1) Observar a ocorrência de CRBs (2); Evocar os CRBs e facilitar o desenvolvimento dos CRBs2; (3) Reforçar CRBs2; (4) Avaliar o efeito do responder do terapeuta sobre o responder do cliente através de perguntas e observações dos efeitos das consequências do seu comportamento sobre os CRBs; e (5) Fornecer interpretações a respeito das contingências que afetam o comportamento do cliente a fim de obter generalização das melhoras obtidas dentro para fora de sessão (Kohlenberg \& Tsai, 1991/ 2001).

A partir da proposta de Kohlenberg e Tsai, em 1991, emergiram diversas pesquisas sobre FAP (Mangabeira, Kanter \& Del Prette, 2012). Como possível consequência do amplo alcance de suas aplicações, uma série de estudos começaram a investigar a efetividade da FAP. Para citar alguns: Busch, Kanter, Callaghan, Baruch, Weeks e Berlin (2009) estudaram um micro processo de análise em que a FAP foi utilizada como o mecanismo de mudança; Oshiro (2011) investigou mecanismos de mudança com o uso de intervenções baseada na FAP, utilizando-se de um delineamento de sujeito único ABAB; Follete, Naugle e Callaghan (1996) discutiram a importância de contribuições teóricas sobre a FAP no relacionamento terapêutico e como este relacionamento poderia exercer função de mecanismo de mudança na terapia; Callaghan, Sumers e Weidman (2003) investigaram a efetividade da FAP no tratamento de clientes com Transtorno da Personalidade Histriônica e Narcisista, entre outros.

Além disso, outros estudos passaram a discutir FAP, alguns associando-a a outras intervenções, tais como Terapia de Aceitação e Compromisso e Terapia Cognitiva de Beck (Ferro-García, 2008; Gaynor \& Lawrence, 2002; Kohlenberg, Kanter, Boling, Parker, \& Tsai, 2002); ou ainda aventando a condução de supervisões baseadas na FAP (Callaghan, 2006; Callaghan, Gregg, Marx, Kohlenberg \& Gifford, 2004; Follete \& Callaghan, 1995) 
Dada a utilidade que a FAP tem demonstrado e a quantidade crescente de publicações, que dificulta os profissionais aplicados a se manterem a par do que tem sido produzido na área, justificam-se trabalhos como este, que objetivam consolidar as publicações de uma determinada área, no caso FAP.

Segundo Skinner (1953/2003), a ciência é uma prática cultural que tem como característica acumular o conhecimento, o que permite avanços e capacita os demais cientistas a irem um pouco mais além. É por meio da comunicação em ciência que se compartilham conhecimentos entre os cientistas e com a sociedade, proporcionando sua inserção cultural e o desenvolvimento tecnológico, características estas que sustentam a atividade científica da pesquisa (Weitzel, 2006; Bronowski, 1997).

Weitzel (2006) sugere que o acesso às informações científicas e apreensão do conhecimento registrado por outros pesquisadores podem viabilizar novos conhecimentos. As publicações de conhecimento científico e as discussões delas derivadas contribuem para as tomadas de decisões em relação aos rumos que uma área de investigação deve seguir. Nesta direção corrobora-se com Witter (2003), que defende que as pesquisas de revisão da produção científica e as meta-análises são instrumentos importantes na ciência, pois seus resultados subsidiam avanços em diferentes áreas do conhecimento.

Para Moura (1997), a análise da produção científica é um procedimento que resulta principalmente em uma quantidade de dados consistentes que permitem aos pesquisadores visualizarem as produções de uma área. Por meio delas, os cientistas podem analisar o estado da produção relacionada ao tema e verificar o grau de replicação de pesquisas, o que, por fim, pode evocar novas estratégias a serem consideradas para a propagação do tema em análise. Portanto, a análise da produção científica assegura o intercâmbio das informações entre os cientistas (Le Coadic, 1994/1996).

Ao se verificar as análises de produção realizadas a respeito de FAP, encontrou-se dois trabalhos: Ferro-García (2008) e Mangabeira et al. (2012), os quais serão descritos a seguir.

A revisão de Ferro-García (2008) analisou os seguintes aspectos em relação à FAP: 1) integração com outras terapias, 2) supervisão e aperfeiçoamento de competências terapêuticas, 3) métodos de avaliação e registro de dados na terapia, 4) efetividade e eficácia, 5) relação entre pensamentos e emoções, e 6) aplicação a problemas clínicos e novos tipos de aplicação a transtornos psiquiátricos específicos.

Os resultados de Ferro-García (2008) mostraram que: a FAP tem sido associada a outras práticas de intervenção, por exemplo, com a Terapia da Aceitação e do Compromisso (ACT) (Callaghan et al., 2004); a FAP tem sido considerada como um instrumento de avaliação do terapeuta e de estabelecimento de aliança terapêutica (Kohlenberg et al., 2002); a FAP tem sido utilizada para intervenções junto a diferentes populações de clientes, por exemplo, com transtorno de personalidade borderline (Vandenberghe, Sousa \& Oliveira, 2005) e com problemas de relacionamentos interpessoais (Callaghan et al., 2004; Kohlenberg, et al., 2002); etc. Diante disso, Ferro-García (2008) concluiu que os procedimentos da FAP podem melhorar outras terapias, além disso, evidenciou a necessidade de pesquisas que consolidem a efetividade ou não da FAP enquanto procedimento de intervenção.

Apesar das contribuições que a revisão de Ferro-García (2008) trouxe para a área, ela não especificou aspectos importantes, tais como o método e os procedimentos que utilizou para seu levantamento, o que impossibilita a comunidade científica identificar a amplitude da amostra analisada.

Outra revisão encontrada na literatura foi elaborada por Mangabeira et al. (2012). Estes autores propuseram caracterizar os estudos a respeito de FAP compreendidos no período de 1990 e 2010, bem como, identificar a evolução do tema e a clareza com a qual os mecanismos de mudança da FAP vinham sendo descritos nas pesquisas analisadas. Diferentemente do estudo de Ferro-García (2008), o qual tinha um maior foco no uso da FAP, a pesquisa de Mangabeira et al. (2012) teve também a preocupação de analisar os aspectos metodológicos da produção da área.

Mangabeira et al. (2012) se propuseram a fazer uma caracterização dos trabalhos levantados. Estes autores realizaram a busca nos seguintes bancos de dados: PubMed, Scientific Electronic Library Online (SciELO), Virtual Health Library - Psychology (VHL-PSI); artigos listados nas referências do site da FAP, http://functionalanalyticp- 
sychotherapy.com; e nos sites Science Direct e ISI Web of Knowledge. E também por meio dos jornais: Journal of Applied Behavior Analysis (JABA), Journal of the Experimental Analysis of Behavior (JEAB), The Behavior Analyst, The Behavior Analyst Today, International Journal of Behavioral Consultation and Therapy (IJBCT) e Journal of Early and Intensive Behavior Intervention (JEIBI). Usaram como palavras-chave os termos Functional Analytic Psychotherapy e literature review, incluíram periódicos em três idiomas: inglês, português e espanhol, coletando desta forma 80 artigos entre 1990 e 2010. Após o levantamento, Mangabeira et al. (2012) analisaram os artigos considerando três características dos estudos: bibliográficas, temáticas e metodológicas.

Os principais resultados em relação às características bibliográficas apresentam que houve um considerável crescimento na quantidade de publicações a respeito da FAP sendo a maioria delas publicações de pesquisadores norte-americanos e tendo como principal autor Robert J. Kohlenberg. Em relação às características temáticas, os resultados encontrados por Mangabeira et al. (2012) apontam uma predominância de estudos versando sobre as contribuições da FAP, intervenções utilizando FAP e características da FAP.

No que se refere às características metodológicas, o estudo de Mangabeira et al. (2012) encontrou diferentes métodos de pesquisa, como por exemplo, métodos que se utilizaram de delineamentos $\mathrm{A} / \mathrm{B} /$ $\mathrm{A}+\mathrm{B}$ ou $\mathrm{A} / \mathrm{A}+\mathrm{B}$, estudos de caso tipo I, II, e III e narrativas de estudos de caso (Mangabeira et al., 2012). Os autores apontam que a variabilidade dos procedimentos de coleta de dados utilizados nas pesquisas analisadas dificultou a comparação dos resultados entre os estudos e a consistência das informações sobre a FAP.

Em suas considerações finais, Mangabeira et al. (2012) afirmaram haver, até 2010, pouca produção empírica sobre FAP e poucos autores dedicados a produzirem estudos na área. Por outro lado, tais autores indicaram que a quantidade de publicações a respeito de FAP apresentou uma aceleração durante as últimas décadas. Tendo isso em vista, o presente estudo tem por objetivo atualizar a análise realizada por Mangabeira et al., considerando a produção científica sobre FAP até o ano de 2013.

\section{Método}

O presente estudo seguiu método semelhante ao utilizado por Mangabeira et al. (2012), tendo algumas poucas diferenças, sendo uma delas o incremento de duas outras bases de dados para consulta: MEDLINE e Lilacs. As bases de dados foram acrescentadas por serem importantes veículos de difusão da área da saúde. Assim, foram consultadas: PubMed, Scientific Electronic Library Online (SciELO), Virtual Health Library - Psychology (VHL-PSI), Medical Literature Analysis and Retrieval Sistema on-line (MEDLINE) e (Literatura Latino-Americana em Ciências da Saúde (Lilacs); artigos listados nas referências do site de FAP http://functionalanalyticpsychotherapy.com; nos sites Science Direct e ISI Web of Knowledge; e também nos jornais: Journal of Applied Behavior Analysis (JABA), Journal of the Experimental Analysis of Behavior (JEAB), The Behavior Analyst, The Behavior Analysis Today, International Journal of Behavioral Consultation and Therapy (IJBCT) e Journal of Early and Intensive Behavior Intervention (JEIBI). As palavras-chave utilizadas foram Psicoterapia Analítica Funcional e a sigla FAP, considerando os idiomas inglês e português. O período de busca compreendeu os anos entre 1990 e 2013.

O resultado encontrado inicialmente foi de 180 publicações. Foram excluídos 134 trabalhos, sendo 80 por já terem sido analisados no estudo de Mangabeira et al. (2012) e 54 por serem duplicados. Portanto, 46 publicações compuseram a presente amostra. Deste universo, 11 artigos datam do período coberto pelo estudo de Mangabeira et al. (2012) mas não foram apresentados por eles, possivelmente por terem sido oriundos das bases de dados adicionais analisadas no presente estudo.

Todos os trabalhos foram analisados sob três aspectos: características bibliográficas, características metodológicas e características temáticas, assim como em Mangabeira et al. (2012). No que se refere as características bibliográficas investigou-se: o ano de publicação, número de autores, nacionalidade dos autores, nome dos autores, país de publicação das revistas e publicações por período. As características metodológicas foram divididas em quatro subcategorias: a) Instrumento de mensuração de dados, b) Estratégia de pesquisa, c) Amostra, sub- 
dividida em: número de participantes e população. Quanto as características temáticas buscou-se: a) Contribuições da FAP; b) Tratamentos que usam a FAP; c) Caracterização da FAP; d) Terapia de Grupo baseado na FAP; e) Integrações de terapias; f) Desenvolvimento de instrumentos baseado na FAP; g) Comparação de ACT com FAP; h) Terapia para crianças e adolescentes baseado na FAP; i) Supervisão baseado na FAP; j) Análise de dados empíricos na FAP; k) Estudos de Eficácia da FAP; 1) Treinando os terapeutas em FAP; m) Terapia de pares baseado na FAP e n) Comparações de CBT ou CT com FAP.

\section{Resultados e discussões}

Com a finalidade de tornar mais clara a exposição dos resultados, a análise dos artigos científicos se inicia pelas características bibliográficas, em seguida são apresentadas as características metodológicas e por último as características temáticas.

A Tabela 1 apresenta a distribuição absoluta e percentual das pesquisas analisadas de acordo com $\mathrm{o}$ ano de publicação.

Analisando a Tabela 1, pode se verificar uma maior quantidade de publicações a respeito de FAP no ano de 2012, quando totalizou 31 artigos. Contudo, os dados apresentados nessa tabela devem ser vistos com cuidado, especialmente o período que compreende 1999 e 2010 que constam apenas os artigos que não foram tratados por Mangabeira et al. (2012).

Recorrendo ao estudo de Mangabeira et al. (2012) e adicionando aos resultados deles os encontrados no presente estudo se chegaria a um total de cinco artigos publicados entre o período de $1990 \mathrm{e}$ 1995, 17 entre 1996 e 2000, 25 entre 2001 e 2005, 44 entre 2006 e 2010 e 35 entre 2011 e 2013. Apesar do total absoluto de artigos publicados no último período ser menor que o anterior, quando relativizado encontra-se uma média de 8,80 e 11,67 artigos/ano, respectivamente. Desta forma, os dados confirmam a tendência de aceleração na quantidade de publicações a respeito de FAP, assim como fora visto em Mangabeira et al. (2012).

Apesar da quantidade relativa de publicações encontradas neste último período (2011 à 2013) ser superior aos períodos anteriores, há de se ter par- cimônia em relação a esta aceleração, dado que no ano de 2012 um único periódico (The International Journal of Behavioral Consultation and Therapy IJBCT) foi responsável pela publicação de 25 dos 35 artigos a respeito de FAP. Tal fato ocorreu em virtude do lançamento de uma edição especial dedicada ao tema. Não há como afirmar que se não fosse essa edição especial o total de artigos teria sido menor, pois é possível que em não havendo a referida edição do IJBCT aqueles artigos teriam sido submetidos a outros períodos; contudo, acredita-se que apesar de possível não seria provável, pois há de se imaginar que alguns desses artigos devem ter sido confeccionados exclusivamente para compor a edição especial.

\section{Tabela 1}

Distribuição Absoluta (F) e Percentual (\%) Referente ao ano de Publicação dos Artigos*

\begin{tabular}{lll}
\hline Ano & F & $\%$ \\
\hline 1999 & 1 & 2,2 \\
2002 & 1 & 2,2 \\
2003 & 1 & 2,2 \\
2005 & 1 & 2,2 \\
2006 & 1 & 2,2 \\
2007 & 1 & 2,2 \\
2008 & 1 & 2,2 \\
2009 & 2 & 4,3 \\
2010 & 2 & 4,3 \\
2011 & 3 & 6,5 \\
2012 & 31 & 67,4 \\
2013 & 1 & 2,2 \\
\hline Total & 46 & 100,0 \\
\hline
\end{tabular}

* Vale ressaltar que os artigos listados entre 1999 e 2010 referem-se apenas àqueles que não foram analisados por Mangabeira et al. (2012).

Outro levantamento feito na presente pesquisa foi identificar o número de autorias presentes nas publicações, se única, quando o artigo conta com um único autor ou múltiplas caracterizada por dois ou mais autores. Tais resultados estão representados na Figura 1.

Como pode se verificar, a autoria múltipla com três pessoas foi a que apresentou maior concentração de artigos, atingindo $37 \%(\mathrm{~N}=17)$ da amostra analisada. Ademais, considerando estudos com 


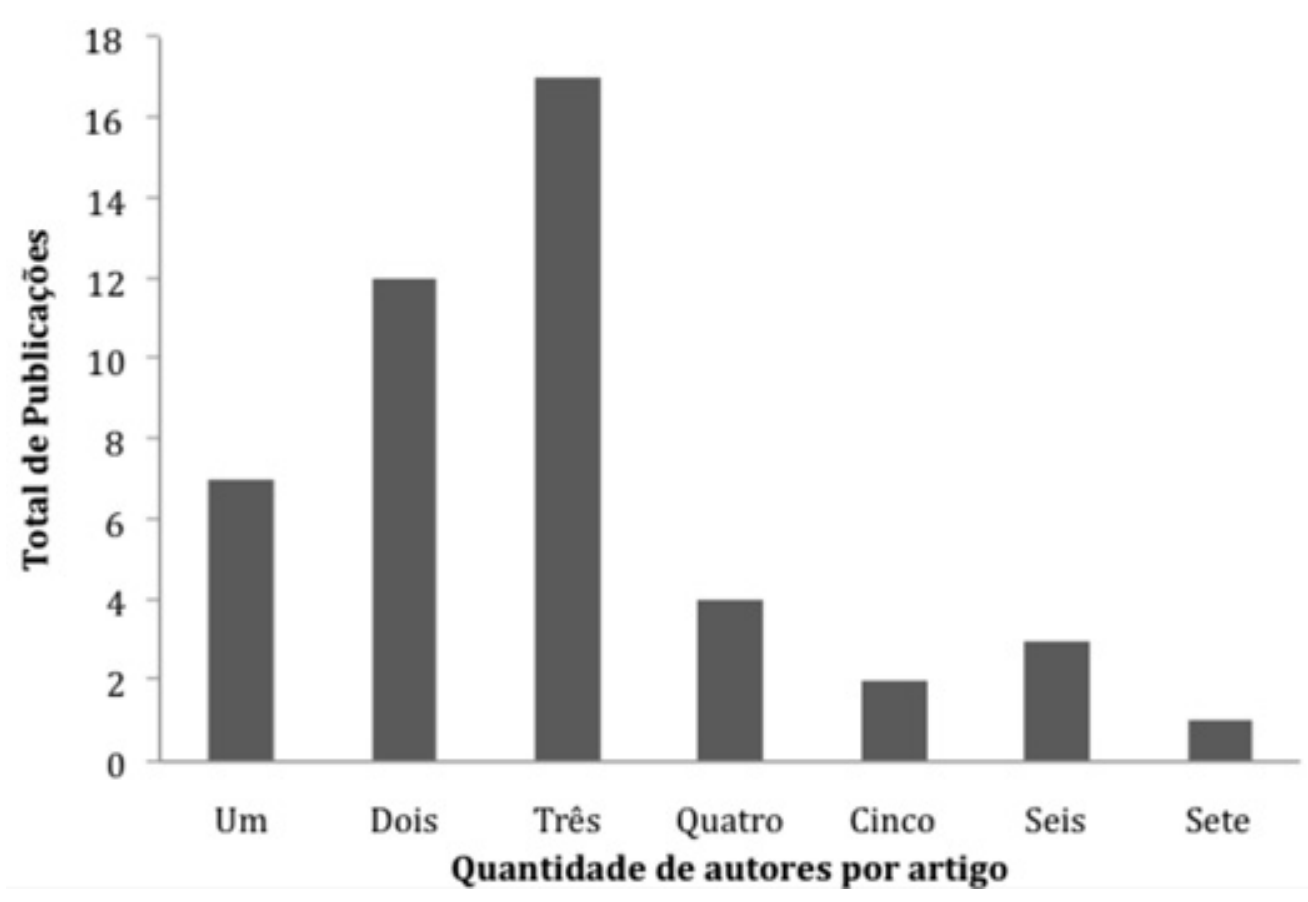

Figura 1. Número de autores dos artigos levantados.

dois ou mais autores, $84,8 \%(\mathrm{~N}=39)$ das pesquisas da presente amostra caracteriza-se por apresentar autoria múltipla.

Analisando a nacionalidade dos autores dos artigos, verifica-se, por meio dos dados da Tabela 2, que os pesquisadores americanos representam $50 \%$ $(\mathrm{N}=67)$ dos autores que publicaram a respeito de FAP na amostra estudada. Os espanhóis compõem o segundo maior grupo, com $19,4 \%(\mathrm{~N}=26)$ dos autores e o Brasil encontra-se em terceiro lugar, com $17,9 \%(\mathrm{~N}=24)$.

Ainda na Tabela 2, verifica-se que os autores que mais publicaram na amostra examinada foram Tsai, Kohlenberg e Ferro-García, sendo os dois primeiros americanos e o último espanhol, cada um como coautor de sete artigos. Em relação a autores brasileiros, destacam-se Oshiro e Vandenberghe, cada qual com três artigos.

Vale ressaltar ainda que em alguns artigos essas múltiplas autorias se caracterizaram por parcerias de pesquisadores de diferentes países. Por exemplo, o estudo The Role of Shaping the Client's Interpretations in Functional Analytic Psychotherapy foi produzido por Paulo Roberto Abreu, Maria Martha Costa Hübner e Fernanda Lucchese (2012), sendo os dois primeiros autores brasileiros e a terceira, uma autora americana; os autores espanhóis Luis Valero-Aguayo e Rafael Ferro-García produziram o estudo Therapeutic Change Processes in Functional Analytic Psychotherapy (2011) em parceria com os americanos Robert T. Kohlenberg e Mavis Tsai; Rodrigo Nunes Xavier e Sonia Beatriz Meyer brasileiros publicaram o artigo Transitional Probability Analysis of Two Child Behavior Analytic Therapy Cases (2012) em parceria com o americano Jonathan William Kanter; as brasileiras Claudia Kami Bastos Oshiro e Sonia Beatriz Meyer e o americano Jonathan William Kanter publicaram A SingleCase Experimental Demonstration of Functional Analytic Psychotherapy with Two Clients with Severe Interpersonal Problems (2012); enquanto que o artigo Functional Analytic Psychotherapy (FAP): A review of publications from 1990 to 2010 (2012) foi produzido pelos brasileiros Victor Mangabeira e Giovana Del Prette em parceria com o americano Jonathan William Kanter.

É provável que a autoria múltipla verificada na Figura 1 decorra da ideia que grupos de pesquisa têm maior potencial de avanço do que trabalhos realizados por pesquisadores únicos, especialmente quando estas múltiplas parcerias ocorrem com pesquisadores de diferentes centros de pesquisa, como foi exemplificado no parágrafo anterior. 
Tabela 2

Distribuição Absoluta (F) e Percentual (\%) Referente ao Número de Publicações de Acordo com a Nacionalidade dos Autores

\begin{tabular}{|c|c|c|}
\hline Americanos & $\mathbf{F}$ & $\%$ \\
\hline Tsai, M. & 7 & 5,2 \\
\hline Kohlenberg, R. T. & 7 & 5,2 \\
\hline Kanter, J. W. & 5 & 3,7 \\
\hline Holman, G., & 3 & 2,2 \\
\hline Bonow, J. T & 2 & 1,5 \\
\hline Callaghan, G. M. & 2 & 1,5 \\
\hline Follette W. C. & 2 & 1,5 \\
\hline Haworth K. & 2 & 1,5 \\
\hline Darrow, S. M. & 2 & 1,5 \\
\hline${ }^{\star}$ Outros & 35 & 26,1 \\
\hline Total & 67 & 50,0 \\
\hline \multicolumn{3}{|l|}{ Espanhóis } \\
\hline Ferro-García, R. F. & 7 & 5,2 \\
\hline Valero-Aguayo, L. V. & 6 & 4,5 \\
\hline Lopez-Bermúdez, M. Á. L. & 4 & 3,0 \\
\hline Vives-Montero, C. V. & 2 & 1,5 \\
\hline${ }^{\star}$ Outros & 7 & 5,2 \\
\hline Total & 26 & 19,4 \\
\hline \multicolumn{3}{|l|}{ Brasileiros } \\
\hline Oshiro, C. K. B. & 3 & 2,2 \\
\hline Vandenberghe, L. & 3 & 2,2 \\
\hline Silveira, J. M. & 2 & 1,5 \\
\hline Meyer, S. B. & 2 & 1,5 \\
\hline Wielenska,R. C & 2 & 1,5 \\
\hline${ }^{\star}$ Outros & 12 & 9,0 \\
\hline Total & 24 & 17,9 \\
\hline \multicolumn{3}{|l|}{ Colombianos } \\
\hline Gómez, M. N. & 2 & 1,5 \\
\hline Gutiérrez, R. V. & 2 & 1,5 \\
\hline Gutiérrez D. G. & 1 & 0,7 \\
\hline Martínez, A. M & 1 & 0,7 \\
\hline Total & 6 & 4,5 \\
\hline Canadenses & & 4,5 \\
\hline Schoendorff, B. & 2 & 1,5 \\
\hline Steinwachs, J. & 2 & 1,5 \\
\hline Total & 4 & 3,0 \\
\hline \multicolumn{3}{|l|}{ Italianos } \\
\hline Cattivelli, R & 1 & 0,7 \\
\hline Tirelli, V. & 1 & 0,7 \\
\hline Berardo, F. & 1 & 0,7 \\
\hline Perin, S. & 1 & 0,7 \\
\hline
\end{tabular}

\begin{tabular}{lll} 
Manduchi,K. & $\mathbf{1}$ & 0,7 \\
\hline Total & $\mathbf{5}$ & $\mathbf{3 , 7}$ \\
\hline Austríaco & & \\
\hline Collis, P. & 1 & 0,7 \\
Total & 1 & 0,7 \\
Britânico & & \\
McClafferty, C & 1 & 0,7 \\
\hline Total & $\mathbf{1}$ & $\mathbf{0 , 7}$ \\
\hline Total & 134 & $\mathbf{1 0 0 , 0}$
\end{tabular}

${ }^{\star}$ Refere-se a autores que tiveram apenas uma publicação na amostra levantada.

Nessa direção, Mangabeira et al. (2012) levantaram a importância da comunicação e do intercâmbio como meios para fortalecer o trabalho científico e melhorar a eficiência dos procedimentos e, consequentemente, do sucesso dos resultados dessas intervenções.

\section{Tabela 3}

Distribuição Absoluta (F) e Percentual (\%) Referente aos Periódicos e suas Nacionalidades em que os Artigos Foram Publicados

\begin{tabular}{lll}
\hline Revistas Americanas & F & $\%$ \\
\hline $\begin{array}{l}\text { International Journal of Behavioral } \\
\text { Consultation and Therapy }\end{array}$ & 25 & 54,3 \\
Psychotherapy & 2 & 4,3 \\
The Analysis of Verbal Behavior & 2 & 4,3 \\
Behavior Modification & 1 & 2,2 \\
Behavior Therapy & 1 & 2,2 \\
Int J Med Psiquiatria & 1 & 2,2 \\
Revistas Espanholas & & \\
Clínica y Salud & 2 & 4,3 \\
Psicotherma & 2 & 4,3 \\
Addictive Disorders. & 1 & 2,2 \\
Apuntes psicol & 1 & 2,2 \\
Associação Espanhola & 1 & 2,2 \\
Neuropsiquiatria & 1 & 2,2 \\
Terapia Psicologica & & \\
Revistas Brasileiras & 2 & 4,3 \\
Psicologia Argumento & 46 & 100,0 \\
Revista Brasileira de Terapia & & 2,2 \\
Comportamental e Cognitiva & & \\
Estudos de Psicologia(Campinas) & & \\
Psicologia: teoria e prática & & \\
\hline Total & & \\
\hline
\end{tabular}


Observa-se na Tabela 3 que a maioria das publicações relacionadas à FAP foram disseminadas pelo periódico americano The International Journal of Behavioral Consultation and Therapy (IJBCT), totalizando 25 artigos. Tal concentração decorre da edição especial que este periódico fez para discutir FAP, como já mencionado anteriormente.

As demais publicações a respeito de FAP se encontram pulverizadas em diferentes revistas, as quais não apresentaram mais que dois artigos a respeito do tema, por ano. Outro aspecto identificado na presente amostra é que revistas americanas e espanholas foram responsáveis pela publicação de 40 artigos, enquanto as brasileiras publicaram apenas seis.

Apesar dos brasileiros serem o terceiro grupo com mais participação em publicações sobre FAP no período analisado, os periódicos nacionais foram preteridos para a publicação, pelo menos se considerarmos a quantidade de artigos publicados nestes veículos.

A pouca predileção aos nossos periódicos pode se dever ao maior fator de impacto que periódicos de língua espanhola e, especialmente, inglesa possuem quando comparados ao fator de impacto de periódicos de língua portuguesa. Tal hipótese ganha força ao se considerar que a publicação de um artigo atende a critérios de avaliação do MEC e/ou, como apontam Dawalibi, Anacleto, Witter, Goulart e Aquino (2013), pela democratização do saber científico. Se forem essas variáveis (ou pelo menos uma delas) que controlam o comportamento de autores no momento de escolha do periódico para publicação, então é forte a tendência a escolher periódicos que apresentem maior fator de impacto, seja pela melhor visibilidade ou pela melhor avaliação que esta escolha propiciaria.

O periódico americano IJBCT parece ser uma referência em publicações a respeito de FAP, não apenas por ter sido encontrada a maior quantidade de artigos desta amostra nele - pois poderia se afirmar que teria sido um evento isolado em decorrência da edição especial, já comentada - mas também por ter sido o segundo periódico que mais publicou artigos sobre FAP no levantamento de Mangabeira et al. (2012). Na amostra desses autores, sete artigos foram oriundos do IJBCT, ficando atrás apenas de The Behavior Analyst Today que publicou oito artigos.
Lançando luz sobre o cenário de periódicos nacionais, pode se dizer que a Revista Brasileira de Terapia Comportamental e Cognitiva (RBTCC) é a revista que mais publica artigos sobre FAP, ao menos se consideradas as últimas duas décadas. Isso porque foram nesses periódicos que se encontrou a maior concentração de artigos publicados no Brasil tanto na presente amostra (dois dos seis artigos publicados, mesma quantidade publicada em Psicologia Argumento), como na amostra analisada por Mangabeira et al. (2012). Além disso, outros dois periódicos apresentaram publicações sobre o tema nas duas amostras, Estudos de Psicologia (Campinas) e Psicologia: Teoria e Prática.

Outro aspecto relacionado a publicação em periódicos nacionais é que, diferente das publicações em periódicos estrangeiros, não houve aceleração na quantidade de publicações, mantendo no triênio analisado uma média de 2 artigos/ano, mesma média encontrada em Mangabeira et al. (2012) no último período (2006-2010).

$\mathrm{O}$ presente artigo faz coro à necessidade de mais publicações a respeito do tema por pesquisadores brasileiros, como defendem Mangabeira et al. (2012). Tal ampliação na quantidade de publicações em língua portuguesa poderia incentivar a discussão e a ampliação das pesquisas no país.

No que se refere aos dados de origem das pesquisas analisadas, verificou-se que 71,7\% (N=33) dos estudos tratam-se de pesquisa de campo ou empírica e $28,3 \%(\mathrm{~N}=13)$ tratam-se de estudos documentais ou teóricos. Dos estudos de campo, 70\% ( $\mathrm{N}=23)$ utilizaram medidas de observações sistemáticas ou assistemáticas para coleta dos dados, enquanto que $30 \%(\mathrm{~N}=10)$ lançaram mão de instrumentos como escalas e questionários padronizados, a despeito das críticas que geralmente analistas do comportamento fazem a este tipo de instrumento.

Há de se mencionar, ainda, a existência de The Functional Analytic Psychotherapy Rating Scale FAPRS, escala criada por estudiosos de FAP, a qual foi utilizada em alguns estudos. Contudo, mesmo com este instrumento tendo sido criado, os resultados encontrados continuam apontando uma falta de padrão de coleta de dados nas pesquisas a respeito de FAP, o que já havia sido afirmado por Mangabeira et al. (2012). Segundo esses autores, 
esta falta de padrão dificulta a comparação de resultados dos estudos e, consequentemente, um corpo de dados consistentes sobre FAP.

\section{Tabela 4}

Distribuição Absoluta (F) e Percentual (\%) Referente ao Número de Publicações de Acordo com a amostra

\begin{tabular}{lll}
\hline Adultos & F & $\%$ \\
\hline 1 participante & 13 & 39,39 \\
2 participantes & 2 & 6,06 \\
3 participantes & 2 & 6,06 \\
5 participantes & 1 & 3,03 \\
6 participantes & 1 & 3,03 \\
16 participantes & 2 & 6,06 \\
22 participantes & 1 & 3,03 \\
76 participantes & 1 & 3,03 \\
104 participantes & 1 & 3,03 \\
193 participantes & 1 & 3,03 \\
303 participantes & 1 & 3,03 \\
544 participantes & 1 & 3,03 \\
582 participantes & 1 & 3,03 \\
\hline Total & 28 & $\mathbf{8 4 , 8 5}$ \\
\hline Crianças ou jovens & & \\
\hline 1 participante & 1 & 3,03 \\
2 participantes & 1 & 3,03 \\
5 participantes & 1 & 3,03 \\
10 participantes & $\mathbf{5 3}$ & 3,03 \\
14 participantes & & 3,03 \\
\hline Total & 100,00 \\
\hline Total & 12 \\
\hline
\end{tabular}

Analisando a Tabela 4, pode-se observar que a maioria dos estudos de campo trabalharam com menos que cinco participantes, sendo predominante os estudos com um único participante $(\mathrm{N}=$ 14). Apesar de provável, não é possível afirmar que esses estudos lançaram mão de um delineamento de sujeito como seu próprio controle, típico da Análise do Comportamento, pois só se verificou a quantidade de participantes o que não é critério definidor deste tipo de delineamento (Johnston \& Pennypacker, 2009; Sidman, 1960/1978).

Chama-se atenção para os estudos que contaram com grande quantidade de participantes $(\mathrm{N}$ > 100), como: Interpersonal Mindfulness Informed by Functional Analytic Psychotherapy: Findings from a Pilot Randomized Trial (Bowen, Haworth, Grow, Tsai \& Kohlenberg, 2012), Therapists' attitudes about and Preferences to Use Relationship Focused Interventions: New Tools to Measure a Critical Component of Functional Analytic Psychotherapy (FAP) (Terry \& Kohlenberg, 2012), Does Acceptance and Relationship Focused Behavior Therapy Contribute to Bupropion Outcomes? A Randomized Controlled Trial of Functional Analytic Psychotherapy and Acceptance and Commitment Therapy for Smoking Cessation (Gifford, Kohlenberg, Hayes, Pierson, Piasecki, Antonuccio, \& Palm, 2011), An Empirical Model of Body Image Disturbance Using Behavioral Principles found in Functional Analytic Psychotherapy and Acceptance and Commitment Therapy (Callaghan et al., 2012) e Reliability and Validity of the Spanish Adaptation of EOSS, Comparing Normal and Clinical Samples (Valero-Aguayo, Ferro-García, Lopez-Bermudez $\&$ Lopez de Huralde, 2012). Seriam eles tentativas de ajustamento dos analistas do comportamento às práticas baseadas em evidência (PBE)? Deixamos a sugestão para que as próximas pesquisas atentem para dois aspectos: primeiro, se há um diálogo entre as pesquisas de FAP e a PBE; segundo, se os estudos de FAP utilizam delineamentos de sujeito como seu próprio controle.

Ainda sobre a Tabela 4, pode se notar que há uma predominância de estudos com participação de adultos nas pesquisas relacionadas à FAP, sendo que dos 33 artigos analisados que contaram com participantes na amostra, $85 \%(\mathrm{~N}=28)$ trabalharam com este tipo de população, enquanto que apenas $15 \%(\mathrm{~N}=5)$ foram estudos com população de crianças e/ou jovens. Tais dados se assemelham aos de Mangabeira et al. (2012), os quais também encontraram a predominância de estudos com adultos (88\% dos artigos analisados) em sua amostra. Neste sentido, não houve mudança na escolha do tipo de população participante das pesquisas na área. Contudo, há a necessidade de estudos com crianças e jovens, para se verificar se os resultados encontrados para adultos são replicáveis nessas outras populações.

Uma última categoria analisada no presente estudo refere-se às características temáticas a respeito de FAP e que estão representadas na Figura 2. 


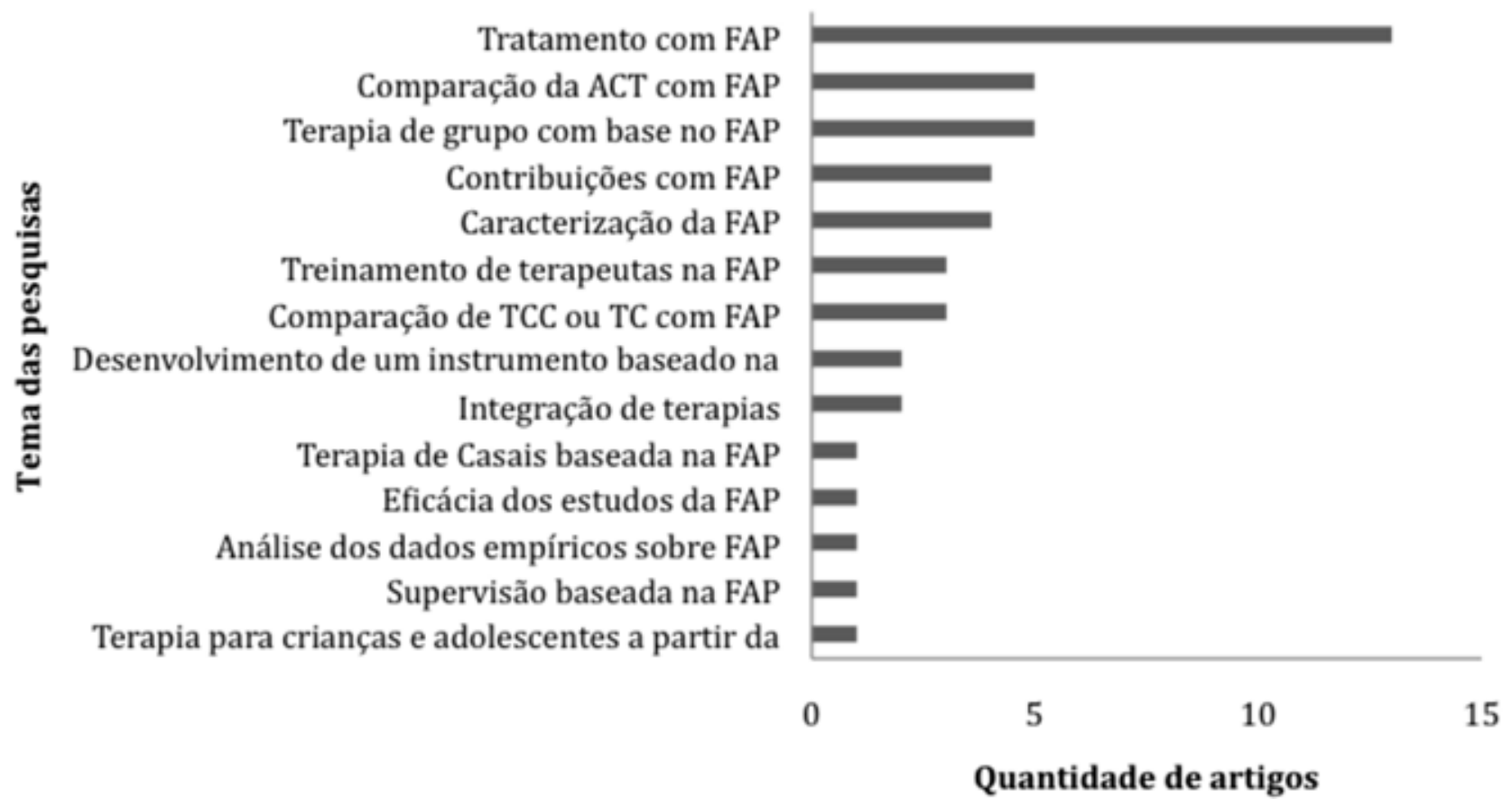

Figura 2: Distribuições dos principais temas encontrados nos estudos de FAP.

Observando a Figura 2, pode se verificar que o tema mais frequente foi tratamento com FAP, totalizando $28,3 \%(\mathrm{~N}=13)$ das pesquisas; seguido pelos estudos que discutiam FAP com Terapia de Aceitação e Compromisso (ACT) e terapia de grupo com base na FAP, cada qual representando 10,9\% $(\mathrm{N}=5)$ do total.

Apesar dos temas mais frequentes no presente levantamento diferirem dos encontrados por Mangabeira et al. (2012), pode se notar que a maioria dos estudos empíricos tiveram como tema principal o tratamento de clientes com FAP e a integração com outras terapias.

\section{Conclusão}

O presente estudo surge da pergunta se teria havido mudança em relação aos estudos de FAP após 2010. Para isso, a pesquisa atual visou caracterizar e discutir a produção científica a respeito de FAP, mais especificamente estendendo o estudo de Mangabeira et al. (2012) para a produção compreendida entre os anos de 2010 e 2013.

De acordo com os resultados encontrados, houve aceleração da produção científica sobre FAP, principalmente no ano de 2012. Tais pesquisas deste ano foram em sua maioria veiculadas pelo periódico americano The International Journal of Behavioral Consultation and Therapy, que publicou um número especial sobre o tema. Também identificou-se que os principais autores das publicações sobre a FAP foram Tsai, Kohlenberg e Ferro-García, sendo os dois primeiros americanos e o terceiro espanhol. Ainda sobre as características bibliográficas, os resultados apontaram que há publicações oriundas de diferentes países como Estados Unidos, Espanha, Brasil, Áustria, Inglaterra, Colômbia, Canadá e Itália.

Dentre as características metodológicas, conclui-se que a maioria dos estudos lançaram mão de medidas de observação para a coleta de dados em detrimento da utilização de escalas e questionários padronizados. Os dados também apontaram que a estratégia de pesquisa de campo ou empírica tem sido amplamente utilizada nos estudos da área, mostrando um aumento relativo deste tipo de estudo em relação a trabalhos teóricos, os quais haviam sido mais frequentes no levantamento de Mangabeira et al. (2012).

Verifica-se que há uma preferência por estudos com um ou poucos participantes, contudo não se pode afirmar que tratam-se de estudos com deline- 
amentos de sujeito como seu próprio controle, pois este aspecto não foi analisado na presente pesquisa, ficando a investigação dos delineamentos utilizados como sugestão para pesquisas futuras.

Ainda em relação a amostra, houve um predomínio de estudos com adultos, assim como no estudo de Mangabeira et al. (2012), permanecendo a necessidade de mais estudos com populações diferentes, como crianças e jovens, visando verificar a extensão dos benefícios da FAP para outras populações.

Os principais temas identificados nos estudos foi tratamento com FAP, comparação de FAP e ACT e terapia de grupo com FAP. Talvez, por serem estes os temas de maior interesse, isso justifique a predominância de estudos de campo ou empírico.

De modo geral, a comparação do presente levantamento com o anterior, de Mangabeira et al. (2012), mostra que houve pequenas mudanças em alguns aspectos, como por exemplo o aumento da proporção de trabalhos empíricos em comparação a trabalhos teóricos, o que é um bom indicativo para a área; enquanto que outros aspectos continuam iguais, como é o caso da manutenção da aceleração na quantidade de publicações na área e o predomínio dos Estados Unidos, seguido por Espanha e Brasil na publicação de pesquisas a respeito de FAP.

Finalizando, a de se deixar registrada algumas limitações deste estudo. Apesar de oferecer uma proposta de produção científica sobre a FAP, não se pode garantir que ele investigou a totalidade de produções a respeito de FAP, pois muitos periódicos e livros podem não ter suas informações carregadas nos bancos de dados consultados. Além disso, cada categoria de análise aqui apresentada contém em si mesma uma grande quantidade de informações que poderia ser melhor desenvolvida e aprofundada - por exemplo uma análise pormenorizada dos delineamentos das pesquisas utilizados.

\section{Referências}

Borges, N. B. (2009). Terapia AnalíticoComportamental: da teoria à prática clínica. In: R. C. Wielenska (Org.), Sobre Comportamento e Cognição: desafios, soluções e questionamentos (Vol. 24, pp. 231-239). Santo André: ESETec.

Bronowski, J. (1997). As origens do conhecimento e da imaginação. $2^{\mathrm{a}}$ ed., Brasília: Editora da UnB. (Publicado originalmente em 1978).

Busch, A. M., Kanter, J. W., Callaghan, G. M., Baruch, D. E., Weeks, C. E., \& Berlin, K. S. (2009). A micro-process analysis of Functional Analytic Psychotherapy's mechanism of change. Behavior Therapy, 40(3), 280-290.

Callaghan, G. M. (2006). Functional Analytic Psychotherapy and Supervision. International Journal of Behavioral and Consultation Therapy, 2(3), 416-431.

Callaghan, G. M., Gregg, J. A., Marx, B. P., Kohlenberg, B. S., \& Gifford, E. (2004). FACT: The utility of an integration of Functional Analytic Psychotherapy and Acceptance and Commitment Therapy to alleviate human suffering. Psychotherapy: Theory, Research, Practice, Training, 41, 195-207.

Callaghan, G. M, Summers, C. J., \& Weidman, M. (2003). The treatment of histrionic and narcissistic personality disorder behaviors: A single-subject demonstration of clinical effectiveness using Functional Analytic Psychotherapy. Journal of Contemporary Psychotherapy, 33, 321-339.

Conte, G. C. S., \& Brandão, M. Z. S. (2012). Eventos a que o clínico analítico-comportamental deve atentar nos encontros iniciais: das vestimentas aos relatos e comportamentos clinicamente relevantes. In: N. B. Borges \& F. A. Cassas (Orgs.), Clínica Analítico Comportamental: aspectos teóricos e práticos (pp. 128-137). Porto Alegre: Artmed.

Dawalibi, N. W., Anacleto, G. M. C., Witter, C., Goulart, R. M. M., \& Aquino, R.C. (2013). Envelhecimento e Qualidade de vida: análise da produção cientifica da SciELO. Revista: Estudos em Psicologia (Campinas), 30(3), 393-403. 
Ferro-García, R. (2008). Recent Studies in Functional Analytic Psychotherapy. International Journal of Behavioral Consultation and Therapy, 4(2), 239-249.

Follette, W. C., \& Callaghan, G. M. (1995). Do as I do, Not as I say: A Behavior-Analytic Approach to Supervision. Professional Psychology: Research and Practice, 26(4), 413-421.

Follete, W. C., Naugle, A. E., \& Callaghan, G. M. (1996). A radical behavior understanding of the therapeutic relationship in effecting change. Behavior Therapy, 27, 623-641.

Gaynor, S. T., \& Lawrence, P. (2002). Complementing CBT for depressed adolescents with Learning through In Vivo Experience (LIVE): Conceptual analysis, treatment description, and feasibility study. Behavioral and Cognitive Psychotherapy, 30, 79-101.

Greben, S. E. (1981). The essence of psychotherapy. British Journal of Psychiatry, 138, 449-455.

Johnston, J. M., \& Pennypacker, H. S. (2009). Strategies and Tactics of Behavioral Research. 3rd ed. New York: Routledge.

Kohlenberg, R. J., Kanter, J. W., Bolling, M. Y., Parker, C. R., \& Tsai, M. (2002). Enhancing Cognitive Therapy for Depression with Functional Analytic Psychotherapy: Treatment Guidelines and Empirical Findings. Cognitive and Behavioral Practice, 9, 213-229.

Kohlenberg, R.J., \& Tsai, M. (2001). Psicoterapia Analítica Funcional: Criando Relações Terapêuticas Intensas e Curativas. Trad. Org. R.R. Kerbauy. Santo André, SP: Esetec. (Trabalho original publicado em 1991).

Mangabeira, V., Kanter, J. W., \& Del Prette, G. (2012). Functional Analytic Psychotherapy (FAP): A review of publications from 1990 to 2010. International Journal of Behavioral Consultation and Therapy, 7(2-3), 78-89.

Moura, E. (1997) ITA- Avaliação da produção científica. In G. P. Witter. (Org). Produção Científica (31-37). Campinas: Átomo.

Oshiro, C. K. B. (2011). Delineamento experimental de caso único: a Psicoterapia Analítica Funcional com dois clientes difíceis. Tese de Doutorado. Universidade de São Paulo. São Paulo

Sidman, M. (1978). Táticas da Pesquisa Científica: avaliação dos dados experimentais na Psicologia.
São Paulo: Brasiliense.

Skinner, B. F. (2003). Ciência e comportamento humano. Trad. J. C. Todorov \& R. Azzi. São Paulo: Edart (trabalho original publicado em 1953).

Taccola, P. A. (2007). A psicoterapia analítico-funcional e relato de sentimentos: Um estudo de caso quase experimental. Dissertação de mestrado, Universidade de São Paulo, São Paulo.

Vandenberghe, L., Sousa, A. C. A., \& Oliveira, J. A. (2005). Treating clients with Borderline Personality Disorder: The impact on the therapist. Communication presented at the 9 th European Congress of Psychology. Granada. Spain.

Weitzel (2006). Fluxo da informação científica. In: D. A. Poblacion, G. P. Witter \& J. F. M. da Silva (Orgs.), Comunicação e produção científica: contexto, indicadores, avaliação (pp. 81-114). São Paulo: Angellara.

Wielenska, R. C. (2012). O papel da relação terapeuta-cliente para a adesão ao tratamento e à mudança comportamental. In: N. B. Borges \& F. A. Cassas (Orgs.), Clínica Analítico Comportamental: aspectos teóricos e práticos (pp. 160-165) Porto Alegre: Artmed.

Witter, G.P. (2003). Professor-estresse: Análise de produção cientifica. Psicologia Escolar e Educacional, 7(1), 33-46.

\section{Referência dos artigos analisados}

Abreu, P. R, Hübner, M. M. C., \& Lucchese, F. (2012). The Role of Shaping the Client's Interpretations in Functional Analytic Psychotherapy. The Analysis of Verbal Behavior, 28, 151-157.

Brandão, M. Z. S. (1999). Terapia comportamental e análise funcional da relação terapêutica: estratégias clínicas para lidar com comportamento de esquiva. Revista Brasileira de Terapia Comportamental e Cognitiva, 1(2), 179-187.

Bonow, J. T., Maragakis, A., \& Follette, W. C. (2012). The Challenge of Developing a Universal Case Conceptualization for Functional Analytic Psychotherapy. International Journal of Behavioral Consultation Therapy, 7(2-3), 2-8.

Bowen, S., Haworth K., Grow, J., Tsai, M., \& Kohlenberg, R. (2012). Interpersonal Mindfulness Informed by Functional Analytic Psychotherapy: Findings from a Pilot 
Randomized Trial. International Journal of Behavioral Consultation Therapy, 7(2-3), 9-15.

Callaghan, G. M., Duenas, J. A., Nadeau, S. E., Darrow, S. M., Merwe, J. V., \& Misko, J. (2012). An Empirical Model of Body Image Disturbance Using Behavioral Principles found in Functional Analytic Psychotherapy, Acceptance and Commitment Therapy. International Journal of Behavioral Consultation Therapy, 7(2-3), 16-24.

Cardoso, L. R. D. (2011). Psicoterapias comportamentais no tratamento da depressão. Psicologia Argumento, 29(67), 479-489.

Carrascoso-López, F. J. (2002). Celos: un caso de aplicación de la psicoterapia analítica funcional/ Jealousy: A case of application of functional analytic psychotherapy. Apuntes psicología. 20(3), 347-368.

Cattivelli, R., Tirelli, V., Berardo, F., \& Perin, S. (2012). Promoting Appropriate Behavior in Daily Life Contexts Using Functional Analytic Psychotherapy in Early-Adolescent Children. International Journal of Behavioral Consultation Therapy, 7(2-3), 25-32.

Collis, P. (2012). An Example of a Hakomi Technique Adapted for Functional Analytic Psychotherapy. International Journal of Behavioral Consultation Therapy, 7(2-3), 33-37.

Darrow, S. M., Dalto, G., \& Follette, W. C. (2012). Equifinality in Functional Analytic Psychotherapy: Different Strokes for Different Folks. International Journal of Behavioral Consultation Therapy, 7(2-3), 38-44.

Ferro-García, R., Lopez-Bermudez, M. A., \& Valero-Aguayo, L. (2012). Treatment of a Disorder of Self through Functional Analytic Psychotherapy. International Journal of Behavioral Consultation Therapy, 7(2-3), 45-51.

Ferro-García, R., Valero-Aguayo, L., \& LopezBermúdez, M. A. (2007). Novedades y aportaciones desde la psicoterapia analítica funcional. Psicothema, 19(3) 452-458.

Ferro-García, R., Valero-Aguayo, L., \& VivesMontero, C. (2006). Application of Functional analytic psychotherapy: Clinical Analysis of Patient with depressive disorder. The Behavior Analyst Today, 7(1), 1-18.
Ferro-García, R., Vives-Montero, C., \& AscanioVelasco, L. (2009). Novedades en el Tratamiento Conductual de Niños y Adolescentes. Clínica y Salud, 20(2) 119-130.

Gifford, E. V., Kohlenberg, B. S., Hayes, S. C., Pierson, H. M., Piasecki, M. P., Antonuccio, D. O., \& Palm, K. M. (2011). Does Acceptance and Relationship Focused Behavior Therapy Contribute to Bupropion Outcomes? A Randomized Controlled Trial of Functional Analytic Psychotherapy and Acceptance and Commitment Therapy for Smoking Cessation. Behavior Therapy, 42, 700-715.

Holman, G., Kohlenberg, R. J., \& Tsai, M. (2012). Development and Preliminary Evaluation of a FAP Protocol: Brief Relationship Enhancement. International Journal of Behavioral Consultation Therapy, 7(2-3), 52-57.

Holman, G., Kohlenberg, R. J., Tsai, M., Haworth, K., Jacobson, E., \& Liu, S. (2012). Functional Analytic Psychotherapy is a Framework for Implementing Evidence-Based Practices: The Example of Integrated Smoking Cessation and Depression Treatment. International Journal of Behavioral Consultation Therapy, 7(2-3), 58-62. Kanter, J. W., Tsai, M., Holman, G., \& Koerner, K. (2012). Preliminary Data from a Randomized Pilot Study of Web-Based Functional Analytic Psychotherapy Therapist Training. Psychotherapy, 50(2), 248-255.

Lopez-Bermúdez, M. A., Ferro-García, R., \& Valero-Aguayo, L. (2010). Intervención en un trastorno depresivo mediante la Psicoterapia Analítica Funcional. Psicothema, 22(1), 92-98.

Maitland, D. W. M., \& Gaynor, S. T. (2012). Promoting Efficacy Research on Functional Analytic Psychotherapy. International Journal of Behavioral Consultation Therapy, 7(2-3) 6371.

Manduchi, K., \& Schoendorff, B. (2012). First Steps in FAP: Experiences of Beginning Functional Analytic Psychotherapy Therapist with an Obsessive-Compulsive Personality Disorder Client. International Journal of Behavioral Consultation Therapy, 7(2-3), 72-77.

Mangabeira, V., Kanter, J., \& Del Prette, G. (2012). Functional Analytic Psychotherapy (FAP): A review of publications from 1990 to 2010. 
International Journal of Behavioral Consultation Therapy, 7(2-3), 78-89.

Martínez-González, J. M. (2010). El efecto de relajación en la percepción de la dinámica familiar de pacientes con trastornos de personalidad. Addictive Disorders, 12(1) 27-32.

McClafferty, C. (2012). Expanding the Cognitive Behavioural Therapy Traditions: An Application of Functional Analytic Psychotherapy Treatment in a Case Study of Depression. International Journal of Behavioral Consultation Therapy, 7(2-3), 90-95.

Mendes, N. A., \& Vandenberghe, L. (2009). O relacionamento terapeuta-cliente no tratamento do transtorno obsessivo compulsivo. Estudos de Psicologia(Campinas), 26(4), 545-552.

Muñoz-Martínez, A., Novoa-Gómez, M., \& VargasGutiérrez, R. (2012). Functional Analytic Psychotherapy (FAP) in Ibero-America: Review of current status and some proposals. International Journal of Behavioral Consultation Therapy, 7(2-3), 96-101.

Newring. K. A. B., \& Wheeler, J. G. (2012). Functional Analytic Psychotherapy with Juveniles who have Committed Sexual Offenses. International Journal of Behavioral Consultation Therapy, 7(2-3), 102-110.

Nieuwsma, J. A., Trivedi, R. B., McDuffie, J., Kronish, L., Benjamin, D., \& Williams Jr., J. W. (2012). Brief Psychotherapy for Depression: A Systematic Review and Meta-Analysis. International Journal of Psychiatry in Medicine, 43(2) 129-151.

Novoa-Gómez, M. M., \& Guzmán-Gutiérrez, D. (2008). Aplicación Clínica de Modelos Terapéuticos No Mediacionales en um Caso de Trastorno Mixto del Afecto. Terapia Psicológica, 26(2), 263-275.

Oshiro, C. K. B., Kanter, J., \& Meyer, S. B. (2012). A Single-Case Experimental Demonstration of Functional Analytic Psychotherapy with Two Clients with Severe Interpersonal Problems. International Journal of Behavioral Consultation Therapy, 7(2-3), 111-116.

Pankey, J. (2012). Functional Analytic Psychotherapy (FAP) for Cluster B Personality Disorders: Creating Meaning, Mattering, and Skills. International Journal of Behavioral Consultation Therapy, 7(2-3), 117-124.

Perón, F., \& Silveira, J. M. (2012). Quando o responder antigo torna-se um comportamento novo: Descrição de um processo de mudança clínica. Psicologia Argumento, 30(70), 491-502.

Pezzato, F. A., Brandão, A. S., \& Oshiro, C. K. B. (2012). Intervenção baseada na psicoterapia Analítica funcional em um caso de transtorno de pânico com agorafobia. Revista Brasileira de Terapia Comportamental e Cognitiva, 15(1), 7484.

Schoendorff, B., \& Steinwachs, J. (2012). Using Functional Analytic Therapy to train therapists in Acceptance and Commitment Therapy, a conceptual and practical framework. International Journal of Behavioral Consultation Therapy, 7(2-3), 72-77.

Terry, C. M., \& Kohlenberg, R. J. (2012). Therapists' Attitudes about and Preferences to Use Relationship Focused Interventions: New Tools to Measure a Critical Component of Functional Analytic Psychotherapy (FAP). International Journal of Behavioral Consultation Therapy, 7(2-3), 138-146.

Tsai, M., Callaghan, G. M., \& Kohlenberg, R. J. (2013). The Use of Awareness, Courage, Therapeutic Love, and Behavioral Interpretation in Functional Analytic Psychotherapy. Psychotherapy, 50(3), 366-370.

Tsai, M., \& Reed, R. (2012). Working In-Vivo with Client Sense of Unlovability. International Journal of Behavioral Consultation Therapy, 7(2-3), 147-150.

Valero-Aguayo, L., Ferro-García, R., Kohlenberg, R. T., \& Tsai, M. (2011). Therapeutic Change Processes in Functional Analytic Psychotherapy. Clínica y Salud, 22(3), 209-221.

Valero-Aguayo, L., Ferro-García, R., LopezBermudez, M. A., \& Selva-Lopez de Huralde, M. A. (2012). Reliability and Validity of the Spanish Adaptation of EOSS, Comparing Normal and Clinical Samples. International Journal of Behavioral Consultation Therapy, 7(2-3), 151-158. 
Vandenberghe, L., \& Ferro, C. L. B. (2005). Terapia de grupo embasada em psicoterapia funcional como abordagem terapêutica para dor crônica: possibilidades e perspectivas. Psicologia: teoria e prática, 7(1), 137-15.

Vandenberghe, L., \& Silveira, J. M. (2012). The Trouble with the Short-Term Therapist-Client Relationship and What Can Be Done About It. International Journal of Behavioral Consultation Therapy, 7(2-3), 159-166.

Virués-Ortega, J., Descalzo-Quero, A., \& VencesláMartínez, J. F. (2003). Aspectos funcionales de la psicoterapia analítico funcional. Revista de la Asociación Española de Neuropsiquiatría, 88, 49-69.

Weeks, C. E., Kanter, J. W, Bonow, J. T., Landes, S. J., \& Busch, A. M. (2012). Translating the Theoretical Into Practical: A Logical Framework of Functional Analytic Psychotherapy Interactions for Research, Training, and Clinical Purposes. Behavior Modification, 36(1) 87- 119.

Wetterneck, C. T., \& Hart, J. M. (2012). Intimacy is a Transdiagnostic Problem for Cognitive Behavior Therapy: Functional Analytical Psychotherapy is a solution. International Journal of Behavioral Consultation Therapy, 7(2-3), 167-176.

Wielenska, R. C., \& Oshiro, C. K. B. (2012). FAP Group Supervision: Reporting Educational Experiences at the University of São Paulo, Brazil. International Journal of Behavioral Consultation Therapy, 7(2-3), 177-181.

Xavier, R. N., Kanter, J. W., \& Meyer, S. B. (2012). Transitional Probability Analysis of Two-Child Behavior Analytic Therapy Cases. International Journal of Behavioral Consultation Therapy, $7(2-3), 182-188$.
Informações do Artigo

Histórico do artigo:

Submetido em: 09/03/2015

Primeira decisão editorial: 30/04/2015

Aceito em: 18/05/2015 